\title{
Shifts in magnitude of reward with humans in the "straightaway"
}

\author{
W. MILES COX and JAY R. WEITZ \\ The William Paterson College of New Jersey, Wayne, New Jersey 07470
}

and

LEWIS R. LIEBERMAN

Columbus College, Columbus, Georgia 31907

\begin{abstract}
Two experiments, one with adults and one with children, were conducted to explore further the phenomenon of incentive contrast effects in a situation more analogous to the instrumental conditioning studies with rats than had previously been employed with humans. Subjects (44 adults and 44 children) rolled a marble along an inclined "straightaway" and into a goal cup at the end. The adult college students earned points toward their course grade for the completion of each trial, while the children were rewarded with tokens which were exchanged for candy. Both adults and children showed improvement in performance as training progressed, but no effect for reward magnitude was obtained, nor did changes in performance occur with shifts in magnitude of reward.
\end{abstract}

Studies involving incentive shifts with animals have shown that subjects shifted from a larger to a smaller reward perform more poorly than subjects which have been trained consistently with the smaller value; conversely, the performance of subjects shifted from a smaller to a larger incentive is not superior to that of subjects which have always received the larger reward. That is, a "negative contrast effect" (NCE) in the absence of a "positive contrast effect" (PCE) has been the typical finding (cf. Black, 1968; Dunham, 1968).

Several studies which are similar in conception to these incentive shift studies of simple instrumental conditioning with animal subjects have been conducted with humans. With a pursuit-rotor task, May and Black (1971), for example, obtained a NCE but no PCE in terms of subjects' persistence in responding following shifts in informative feedback with regard to the quality of their performance. The same results (a NCE, no PCE) were obtained by Calef, Calef, Bone, Thomas, and Fox (1971) with a star-tracing task in which subjects received points toward their course grade for each segment successfully completed in a given interval of time. In the case of human probability learning in which subjects guess which stimulus event will occur on each trial, shifts in the magnitude of reward received for a correct response have, however, produced conflicting results. In terms of the frequency with which the stimulus events are guessed, a NCE but no PCE; neither a NCE nor a PCE; and both a PCE and a NCE have all been reported (cf. Chapman and Halpern, 1969; Halpern, Schwartz, \& Chapman, 1968).

This paper is sponsored by Judson S. Brown who takes full editorial responsibility for its contents. Requests for reprints should be sent to W. Miles Cox, who is now at the Department of Medical Psychology, University of Oregon Health Sciences Center, Portland, Oregon 97201.
The present experiments sought to clarify an 1 to extend the research on contrast effects with human subjects by using a procedure which was more nearly analogous to the simple conditioning experiments with animals than the procedures with human subjects which had been previously employed. Thus, not only was the experimental task designed to resemble the instrumental running response of the rat in the "straightaway," but in the same way that magnitude of the reward which the rat receives in the goalbox is typically not dependent upon the "quality" or "speed" of its performance, no such contingency held in the present procedure.

\section{METHOD}

\section{Experiment 1}

The subjects in Experiment 1 were 44 adults (16 males, 28 females; mean age $=26.27$ years, $S D=9.22$ ) who were enrolled in a course in general psychology at The William Paterson College. The apparatus consisted of an enclosed wooden "straightaway" whose interior was $163.84 \mathrm{~cm}$ in length, $12.70 \mathrm{~cm}$ wide, with sides $5.72 \mathrm{~cm}$ high. One end of the apparatus was designated the "start area," and the other end was the "goal area" where there was a "goal cup" whose rim was affixed flush with the end of the floor of the apparatus. Further, the "goal area" was elevated $5.72 \mathrm{~cm}$ above the table on which the apparatus rested so that it was in a slightly inclined position, and wooden obstructions in the shapes of parallelograms and rectangles which were 5.08 to $15.24 \mathrm{~cm}$ in length, 1.91 to $2.54 \mathrm{~cm}$ wide, and approximately $2.00 \mathrm{~cm}$ high were affixed to the floor of the apparatus at various points along its interior.

Each subject was tested individually in an $8 \times 10 \mathrm{ft}$. experimental room, and the experimental procedure was explained to the subjects through written instructions. The subjects' task was to roll a marble along the length of the apparatus from the start area to the goal area and into the goal cup at the end, but rather than touching the marble directly, the subjects were permitted to roll it along only with the aid of a pipe cleaner. At the beginning of each trial, the experimenter said, "Ready, go!" and with the aid of a stopwatch the subject 


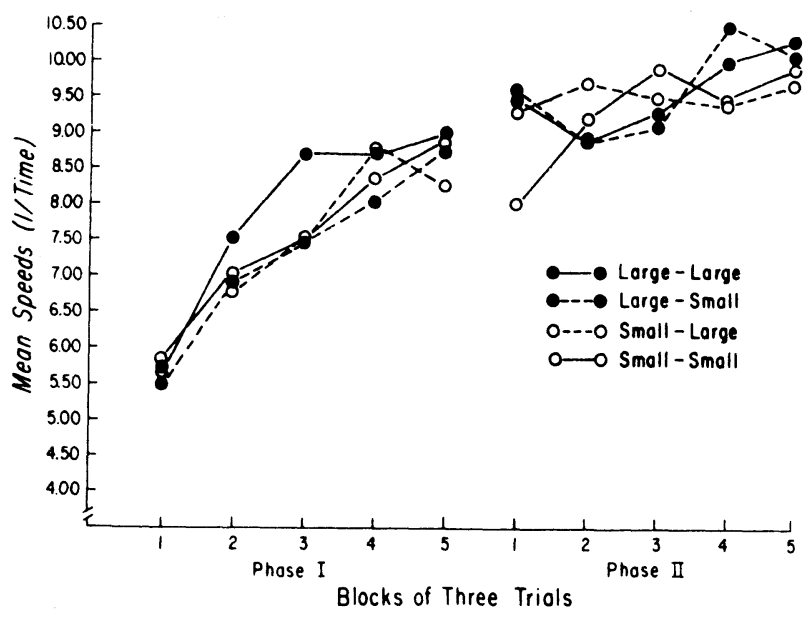

Figure 1. Mean response speeds of the four groups of adults in Experiment 1 during the preshift and postshift phases.

was timed from the "Go" signal until the marble dropped into the goal cup. Subjects performed the task for a total of 30 trials, although they were informed only they would have many opportunities to roll the marble into the goal cup, the total of which would take about $30 \mathrm{~min}$ to complete. Before the experiment began, the subjects were also informed that if they became fatigued at any time before the completion of the trials, they would be able to rest between trials.

The subjects earned points for the completion of each trial which were applied toward their final grade in the course. Accordingly, before the beginning of each trial, the experimenter placed into the goal cup a folded slip of paper which indicated the number of points which the subject would receive for that trial, and once the subject had rolled the marble into the cup, he or she unfolded the paper to determine the number of points for that trial and then handed the slip of paper to the experimenter to record. (A maximum of several hundred points could be accumulated in the course through quizzes and participation in experiments.)

Subjects specifically were given no information with regard to the number of points they would receive for performing the task; nor were they told whether the number of points received would be a function of the speed or "quality" of their performance or if the number of points would remain the same throughout the experiment. If the subject asked questions regarding these matters, the experimenter simply indicated that he could not answer these questions but briefly reiterated the task which was expected of the subject. To determine the number of points that each subject actually would receive, subjects were assigned randomly to one of four groups: (1) Group Large-Large (L-L) consistently received a large reward (10 points per trial) throughout the 30 trials of the experiment, while (2) Group Small-Small (S-S) always received one point. (3) Groups Small-Large (S-L) and (4) Large-Small (L-S), on the other hand, were shifted from the small to the large reward, or vice versa, after 15 trials. 1

\section{Experiment 2}

In order to determine if shifts in magnitude of reward with the present procedure would produce different effects with different age groups like those which have previously been reported (cf. Weinstein, 1972), a second experiment was conducted with children. The subjects ( 20 males and 24 females) who ranged in age from 6 to 13 years $($ mean $=9.39, \mathrm{SD}=1.79)$ were volunteers from the Teaneck Summer Arts and Crafts Program at the Teaneck Town House, Teaneck, New Jersey. Eleven subjects were randomly assigned to each of four groups, the same as those in Experiment 1. The apparatus and procedure were also the same as those of Experiment 1 except for the type of reward employed. In this case, prior to each trial, tokens (buttons) were placed into the goal cup which was then covered to prevent the subject from seeing how many buttons were so placed. At the end of a trial, the subject removed the tokens which he or she then retained until 30 tokens had been accumulated, at which time they were exchanged for a miniature $(1 / 4 \mathrm{Oz})$ chocolate candy bar. (Subjects were not allowed to eat the candy until all of their trials had been completed.) In Experiment 2, the large reward was varied randomly among seven, eight, and nine tokens, while the small reward was varied randomly among one, two, and three tokens, and the experimental groups received the appropriate shift in reward after 15 trials. A total of 120 tokens, which were exchanged for four candy bars, was earned by the large-reward subjects during each of the two phases of the experiment, while the small-reward subjects earned a total of 30 points, or one candy bar, during each phase.

\section{RESULTS AND DISCUSSION}

Prior to analysis, all time scores were converted into speeds ( $1 /$ time $)$, and trials were collapsed into blocks of three trials each. These response speeds are presented in Figures 1 and 2 for the adults and for the children, respectively. During the preshift phase, both the adults and the children showed improvement in performance as training progressed, $F(4,160)=30.74$ and 22.63, $\mathrm{p}<.001$, respectively. Among the adults, Group LL appears to have responded more rapidly than the other three groups which do not appear to differ from each other, but statistical analysis failed to confirm any reliable group differences, $\mathrm{F}<1.0$. Among the children, the two sinall reward groups, considered together, appear to have responded somewhat more rapidly than the two large reward groups, but analysis revealed that the children too did not perform reliably differently for the two magnitudes of reward, $\mathrm{F}<1.0$.

During the postshift phase, the trends in performance of the four groups suggest that there was some additional improvement with the additional training for the adults as well as for the children, and statistical analysis confirmed that the changes in performance with

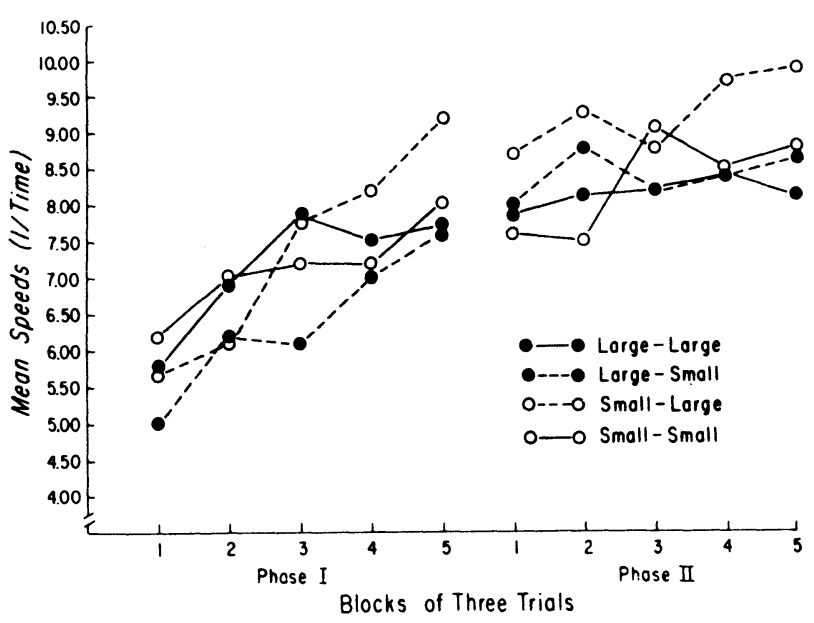

Figure 2. Mean response speeds of the four groups of children in Experiment 2 during the preshift and postshift phases. 
trials was reliable for both age groups, $F(4,160)=3.11$ and 2.53, $\mathrm{p}<.05$, respectively. Among neither the adults nor the children, however, was the performance of the nonshifted groups dependent on reward magnitude. Inspection of the performance of the shifted groups indicates further that among the adults, Group L-S did not perform more slowly than Group SS and neither did Group S-L perform more rapidly than Group L-L. Statistical analysis of the aduits' postshift performance confirmed that there were no reliable group differences, $\mathrm{F}<1.0$, nor were the magnitudes of the changes in performance with trials different for the four groups, as reflected by a nonreliable groups-by-trials interaction, $F(12,160)=1.13, \mathrm{p}>.20$.

Among the children, Group L-S likewise did not "undershoot" the performance of the small reward control group. Although Group S-L responded consistently more rapidly during the postshift phase than Group L-L, it should be noted that this trend was already apparent at the conclusion of preshift training and thus it should probably best be interpreted as reflecting an error in sampling. Analysis of variance performed on the postshift response speeds of the children indicated that they too showed neither reliable group differences, $F(3,40)=1.12, p>.20$, nor a reliable groups-by-trials interaction, $F(12,160)=1.04, p>.20$. In summary, then, the present results provide no evidence whatsoever either for an effect for magnitude of reward or for changes in performance accompanying shift in reward magnitude.

There are several possible explanations to account for the present negative findings. Perhaps the discrepancy between the large and small reward was not large enough for a reward magnitude effect to be demonstrated. Perhaps it did not seem credible to the subjects that points earned for the marble-rolling task actually would be applied toward their course grade or that the tokens would be exchanged for candy. Or even given the credibility of the experimental procedure, perhaps the primary reinforcers were temporally too far removed from the "points" and the "tokens" for the latter to function as effective secondary reinforcers. Yet, these explanations do not seem adequate to account for the discrepancy between the present results and those of the Calef et al. (1971) study in which the nature of the reinforcement as well as its magnitude was similar to that employed in the present Experiment 1.
It should be recalled that in the prior reward-shift studies employing human subjects which obtained reward magnitude and contrast effects, there was an actual or an implied contingency between the speed or accuracy of the subjects' responses and the magnitude of the reward which they received. Thus, another possible explanation for the present findings is that due to the absence of this contingency in the present procedure, subjects did not respond more rapidly for the large reward simply because it was not expedient for them to do so. The implication of this explanation, moreover, is that in an instrumental learning situation such as the present one, the human subject may not be susceptible to the simple conditioning processes which affect the behavior of the rat in such a situation. That is, the rat responds more rapidly for a large reward than for a small reward not because the larger reward is contingent upon its faster performance but because greater "excitation" or "incentive motivation" for that larger reward has been conditioned. Due to the paucity of the experimental results with regard to shifts in magnitude of reward with the human, we wish to hold choice among these alternative explanations in abeyance until more extensive data are available with our present procedure.

\section{REFERENCES}

Black, R. W. Shifts in magnitude of reward and contrast effects in instrumental and selective learning: $A$ reinterpretation. Psychological Review, 1968, 75, 114-126.

Calef, R. S., Calef, R. A. A., Bone, R. N., Thomas, T. A., \& Fox P. A. A human analogue of discrimination contrast. Psychonomic Science, 1971, 23, 191-192.

Chapman, C. R., and Halpern, J. Positive contrast effects as a function of method of incentive presentation. Journal of Experimental Psy chology, 1969, 80, 548-552.

Dunham, P. J. Contrasted conditions of reinforcement: A selected critique. Psy chological Bulletin, 1968, 69, 295-315.

Halpern, J., Schwartz, J. A., \& Chapman, R. Simultaneous and successive contrast effects in human-probability learning. Journal of Experimental Psy chology, 1968, 77, 581-586.

May, R. J., Jr., \& Black, R. W. Persistence of responding on a perceptual-motor task following shifts in informative feedback. Psychonomic Science, 1971, 72, 233-234.

Weinstein, L. Magnitude of human incentive contrast as a function of amount of training and age. Psychonomic Science, 1972, 26, 91-94.

\section{NOTE}

1. After the experiment had been conducted, subjects in Groups S-S, L-S, and S-L had the opportunity to earn additional points so that all participants in the experiment, in effect, had an equal number of points applied to their grades.

(Received for publication February 27, 1975.) 